\title{
A Grid Routing Scheme Considering Node Lifetime in Ubiquitous Sensor Networks*
}

\author{
Sangjoon Park ${ }^{1}$, Sok-Pal Cho ${ }^{2}$, and Byunggi Kim ${ }^{3}$ \\ ${ }^{1}$ Info. \& Media Tech. Institute, Soongsil Univ., Korea \\ ${ }^{2}$ Dept. of C \& Comm. Eng., Sungkyul Univ., Korea \\ ${ }^{3}$ School of Computing, Soongsil Univ., Korea \\ lubimia@hanmail.net
}

\begin{abstract}
Ubiquitous Sensor Networks (USN) environment is composed of dense nodes of Ad-Hoc network structure which has small size node, low power and low calculation ability. Sensor nodes have constraints in operation which small size and distribution features so that they should be operated using low power. In this paper we propose a grid computing scheme to solve limited energy problems which can maintain the energy consumption as a same level though measuring remain energy with analyzing energy consumption of nodes. We confirm the improvement of survival time of entire nodes and less variation of topology in case of our proposed scheme through simulation.
\end{abstract}

\section{Introduction}

USN is a network that forms ad-hoc network structure, in which small sensor nodes clustered together monitor surrounding environment and gather information. The information can be gathered in various fields from general environments in daily life to specific environments hardly controlled by human. Small sensor nodes sense, calculate, store, communicate, and transmit the information. The need for USN is being increased from day to day because USN takes charge of an important role, which provides very useful information in important and serious situations of daily lives, military situations, or disasters on the basis of wireless sensor communication techniques [1]. In sensor networks, the difference between required traffics or between applied methods occurs depending on characteristics of sensor nodes or its application fields. Therefore, new protocol, which is different from existing protocols of cellular or ad-hoc network, is needed [3]. There are also many limitations, which are driven by characteristics that sensor nodes use small amount of electricity to process its tasks due to its small size and its distributional characteristics. Various approaches have suggested many methods considering usage of limited electricity [4][5], but most of the approaches may not consider effectiveness of total nodes. Communication in USN environment normally uses broadcasting method [7], and almost instances of the routing method usually use Flooding or Gossiping method. However, energy waste problem can occur on the part that is duplicated or excluded

* This work was supported by the Korea Research Foundation Grant (KRF-2004-005-D00147). 
in the path establishment. It can be a serious problem depending on selection of routing paths. Another problem of existing methods is that excessive energy consumption or accuracy depreciation in the traffic transmission can also occur [6]. In addition, traffic overload can be concentrated over specific nodes in many cases and it makes some nodes lose their ability. Handling these problems increases energy consumption and it creates another problem that the accuracy of traffic transmission is decreased. In this paper, we suggest grid algorithm-based routing method as a solution of these problems. This method is different from existing methods using grid points [6][8], because it uses transmission radius of sensor nodes when grids are produced. Our method conserves significant amount of energy and keeps regularly energy consumption using a simple measurement of remaining amount of energy, and it is not difficult to set transmission paths.

The remainder of this paper is structured as follows. In section 2, we suggest grid algorithm which uses the energy consumption model and energy level concept described in section 2 . In section 3 , we analyze and verify our grid algorithm using performance assessment. Finally, section 4 shows the conclusion.

\section{Grid-Based Routing Algorithm}

\subsection{Energy Level of Sensor Node}

Basically, the transmission method of sensor nodes uses a broadcasting method which transmits traffic to all surrounding nodes. The broadcasting method is shown in Fig. 1. To use a flooding or another routing method creates energy wasting problem during the traffic transmission process because of extended directions, increased traffic, or wrong routing path selection [9]. For reduction of the energy waste due to wrong routing, this grid algorithm proposes a new concept, transmission level. It is a transmission method that transmits traffic through the corresponding transmission level by setting transmission level to each node from sink to destination depending on its transmission radius. In this method, each node receives the information in their transmission level, and sends it to another node in previous transmission level. It can minimize the waste of transmission traffic because of the confusion of direction. For this transmission level, we add 2 bit field. The value of the field can be 4 different values: 00, 01, 10, and 11. As shown in Fig. 2, transmission level classifies the radius that a node's transmission can be reached.

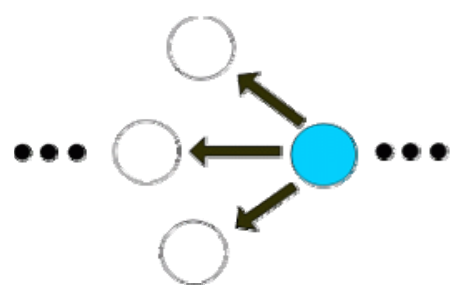

Fig. 1. Node broadcasting 


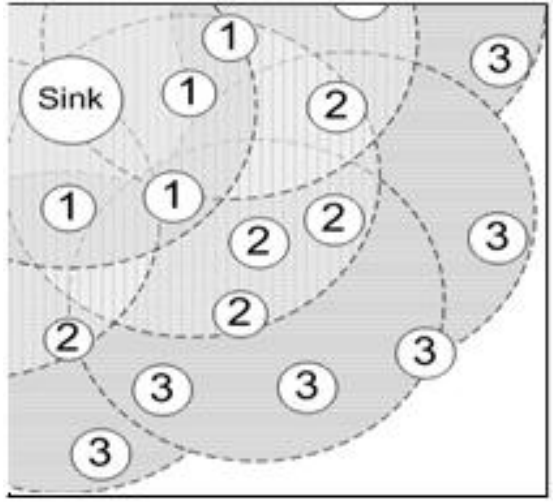

Fig. 2. Generating process of transmission level

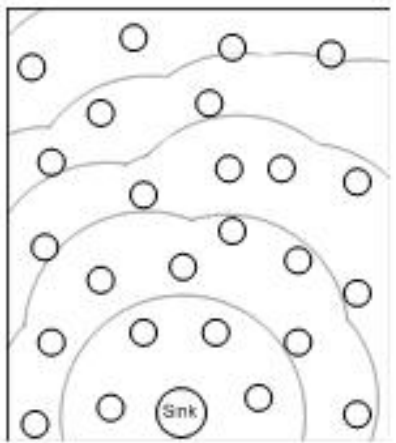

(a)

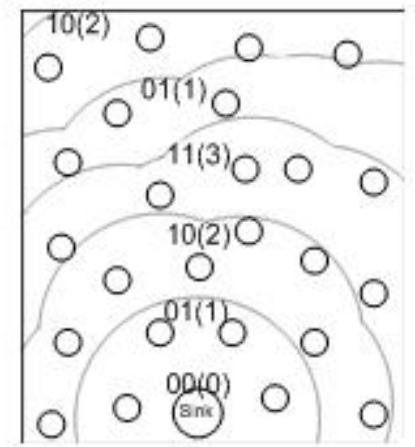

(b)

Fig. 3. Transmission rage of sink

As shown in Fig. 2, every node received the initial routing information in transmission radius of sink gets $01(1)$ as the value of transmission level. Then, new nodes received the routing information from these nodes get 10(2), and next nodes get 11(3), and so on. However, the transmission level provides only 2 bits so that the value must be repeated as a loop structure: $01,10,11,01,10,11,01 \ldots$ The value 00 is used to distinguish sink from others. Fig. 3(a) shows previous state that transmission level is not generated yet, and Fig. 3(b) shows latter state that the generation of transmission level has been finished. In Fig. 3(a), a transmission radius is shown around sink. Each node has its own transmission radius, and the radius is synchronous with others. Because of the synchronous characteristics, it is possible to communicate with each other from both directions. The decision of transmission level is achieved by Flooding method, and it has been decided in order during the period in which the routing process of path is being processed. The value of transmission level of a node is decided by using the information received from its surrounding nodes. After the decision of transmission level, the traffic transmission from the destination to sink is carried out in 


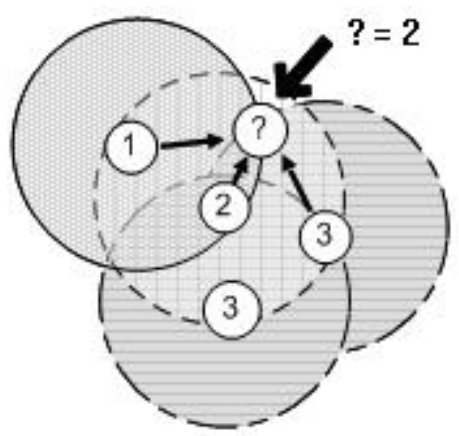

Fig. 4. Transmission level decision in case of that a new node is added

reverse order. When traffic is transmitted through transmission level, the only thing we have to consider is that the transmission level which has the value 01(1) or 11(3) is repeated again. Above transmission level is also adaptable to the change of network. If a new node is added or deleted, its transmission level can be decided by transmitting the information that shows the relation with surrounding nodes. This method is much easier to adapt to topology than other existing methods, and Fig. 4 shows it as a picture. In Fig. 4, we can see that a new node receives signals of all transmission levels $(1,2,3)$. In this case, lower or higher level is recognized as its higher or its lower level. Accordingly, the medium value, transmission level 2, is the transmission level of the new node. Almost transmission levels have values which is one-level higher than the smallest number (1).

\subsection{Dynamic ID Generation for Node Identification}

In Broadcast routing method, a sender node sends traffic to all nodes in its transmission radius. Because of this transmitting method, unnecessary energy consumption can takes place. Dynamic ID generation method suggested in this paper creates the minimum number of identifiers, which can distinguish it from other nodes only within the node's transmission radius. This method is different from others because the setting of IDs is executed only in initial routing state or in case of topology change. The method also minimizes the number of interruptions or conflicts using the methods as follows: ID allocation method randomized by arrival time, re-adjustment process, and channel distinguish method. At first, if a node has received information from sink or another node, then the node sets a random value as its unique ID values. The random value depends on the time information that is received. The sender node transmits information to all nodes in its transmission radius, and waits information since a setting delay time which the receiver nodes send back. The delay time depending on ID would spend twice time as much as time for transmission plus additional time for transmission time block, which makes receiver nodes send information only within its transmission time that is suitable for its ID. Each node can send information to receiver nodes within its transmission time depending on its ID, and the nodes received the information set its ID as 1 from existing value 0 . Setting ID as 1 is used for conflict prevention to confirm that there is any existence of same ID. If a sender node has been waiting for delay time same as the total ID and then 
duplication occurred, the node ask the node which ID is occurred the duplication readjustment for duplication ID. The signal in this process is divided and used for multichannel, and it prevents signal intervention among the same level nodes. It takes a role that there are not any intervention to each other by using a different channel for each receiver node. ID adjustment for other nodes which transmission level is higher than the receiver node is only needed for this process. It's because there is no intervention if we prevent ID conflicts of higher nodes due to the difference of transmission radius. Fig. 5 shows this dynamic ID allocation process.

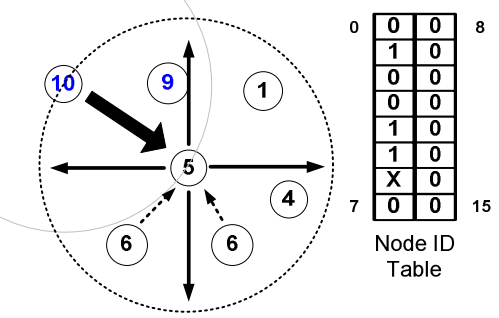

Fig. 5. Dynamic ID allocation process of a node

In Fig. 5, ID 9 and ID 10 are located in the black circle area and represent higher transmission level. The fifth node 5 is received signal from transmission ID 10, and then sends signal to every node in its transmission radius to check the internal conflict. According to the time, node 5 has been received signal and fills the table from the nodes in its transmission radius. After marking the IDs of node 1, 4, and 5 (itself), node 5 is received ACKs from two nodes 6 , and it notices that there is duplication of ID 6 . In this case, node 5 continuously checks information about other nodes. After finishing the process, it asks to accomplish re-adjustment process for the nodes which have the same ID. This method uses TDMS per each node and uses the structure of SMAC and EAR that UCLA proposed [10]. Even though a new node is added, the new node is available to set its ID by receiving information from surrounding nodes.
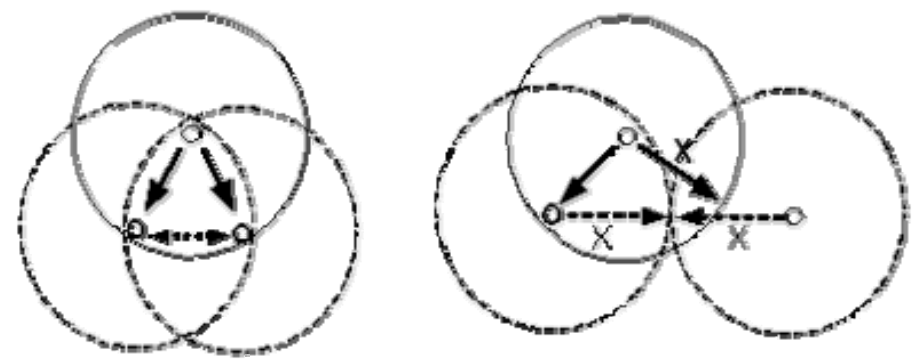

Fig. 6. Conflict consideration for the difference of transmission rage among nodes 
These dynamic ID values is needed to distinguish among others in each node's transmission radius, therefore, there can be more than two nodes which have same ID but their transmission radius is not duplicated. As you can see in Fig. 6, the left part shows that each node should have different ID, and the right part shows that two nodes should have different IDs, but the node which is located the right hand side doesn't need to have different ID with the other nodes because it is in different transmission radius which is not duplicated with the other's. As a result, this method has an advantage that it needs small storage because we use same IDs to different nodes if they are not in the same transmission radius or even though they have the same transmission level if they are far from each other, so they can't have any effect on each other.

\subsection{Grid Table for the Sensor Node}

Grid based sensor node routing suggested by this paper needs signals which control electric power of sensor nodes. The signals take a role that transmits node information to RTS/CTS and keeps energy remaining grid uniformly by the grid algorithm which uses corresponding information. The new signal information that is transferred with existing RTS/CTS information for grid algorithm-based routing is followed (Fig. 7).

- Transmission level of sender/receiver nodes

- Dynamic IDs for sender/receiver nodes

- Energy level of sender nodes

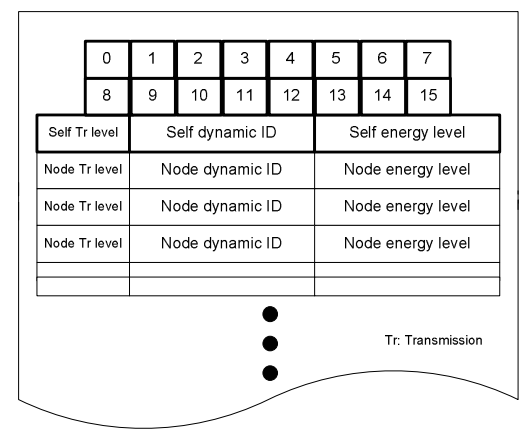

Fig. 7. Storage structure of grid table

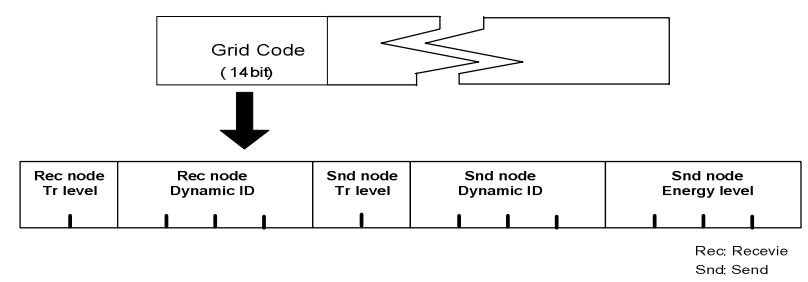

Fig. 8. Grid codes for grid routing 
Fig. 7 shows grid table which is used for each sensor nodes by using RTS/CTS node information like this. In the table structure of Fig. 8, the upper part of $1 \sim 15$ is 1 bit and a part that checks dynamic ID conflicts within its transmission radius. Grid table for transmission consists of transmission levels for reachable nodes, IDs for corresponding nodes, and energy levels. If there is ID information received from surrounding nodes, the table sets the value as 1 . Below the information, the table stores received information from the nodes of lower transmission level, and it forms grid table structure which is used for real traffic transmission.

\subsection{Grid-Based Transmission}

The most important parts of traffic transmission in sensor network are effectiveness and speediness. To reduce energy consumption in traffic transmission, transmission should be transferred in maximum accuracy. In this section, we propose an approach that reduces energy consumption in transmission and increases effectiveness of total nodes. This grid based routing approach is described using transmission level and energy level we've already described. We suppose that sensor network structure for grid algorithm needs these assumptions:

- Transmission among nodes is always bi-directional (transmission radius is same).

- List information depended on energy level is kept in grid table.

- Information in grid table is changed by energy level, update occurs through RTS/CTS information.

- Basically, network considers periodical Sleep states depending on S-MAC.

- The node which remaining energy amount has fallen less than critical value doesn't take part in traffic transmission anymore.

Through these assumptions, grid-based routing algorithm is as follows:

(1) Using routing algorithm, we create transmission level, dynamic ID, and grid table.

(2) If there is traffic transmission, then we adjust the values of grid table using RTS/CTS transmission and execute grid computing.

(3) Grid computing takes place into the direction which transmission level is lower and which energy level is higher.

(4) If energy levels of nodes are same, then choose any arbitrary node and transfer traffic because the result is same although we choose any node.

(5) In case of that we can't send information to a node of low energy level, we send it through the path which nodes have same transmission level. In this case, we also choose the transmission through the node which has the highest energy level, and the path which uses the smallest energy consumption.

(6) During the process (2) to (5), information of grid table is updated through RTS/CTS.

- In traffic transmission, information of grid table is used, and real amount of remaining energy is updated through CTS. This information is used for resource for next grid computing.

- Because there is a limitation in the usage of buffer which is stored in grid table, information of the nodes which have more higher energy level should be stored in grid table among a lot of received information. 
(7) If the remaining energy level of a sensor node has fallen under the critical value, the node neither can stay in grid table nor can be used for traffic transmission. The node also is restricted the change of network topology through signal transmission.

Energy level used in this algorithm makes to allow grid change by the remaining amount of energy, and to move the remaining amount of energy level for each sensor node to standardized value. Our main purpose is also not to change the construction of topology for sensor node itself. Due to these advantages, the use of uniformed energy usage is possible through the whole sensor network and we can use it with existing routing methods. In addition, in treatment of traffic among nodes, we can increase lifetime of sensor nodes and reduce uncertainty of traffic transmission when energy exhaustion is taken place because of lack of resource in some nodes.

\section{Performance Evaluations}

Simulation environment for grid based routing method is an environment which is based on IEEE 802.11 MAC structure. We had observed the increase of lifetime based on grid algorithm by distribution 100 nodes in an area of $100 \times 100 \mathrm{~m}^{2}$. We also look into the energy usage of nodes through each grid table. As we observed the parameters in the beginning of this simulation, the initial energy value of each node was $0.5 \mathrm{~J}$. Grid routing algorithm had processed by measuring real energy consumption and by considering the amount of traffic transmission and the continuance time of each mode. The transmission radius for each node is 10 meters, traffic length which is really transmitted is 400 bytes, and information delivery for transmission consists of 16 bytes. The information for simulation parameters is shown in Table 1 [2].

Table 1. Simulation parameters

\begin{tabular}{|c|c|}
\hline Item & Application \\
\hline Network area & $(100 \mathrm{~m}, 100 \mathrm{~m})$ \\
\hline Node number & 100 \\
\hline Packet size & 400 Byte \\
\hline Grid Packet size & $16 \sim 19 \mathrm{Byte}$ \\
\hline Radio Range & $20 \mathrm{~m}$ \\
\hline Transmit mode EC & $24 \mathrm{~mW}$ \\
\hline Receive, Idle mode EC & $13 \mathrm{~mW}$ \\
\hline Sleep mode EC & $15 \mathrm{uW}$ \\
\hline Node Initial Energy & $0.5 \mathrm{~J}$ \\
\hline
\end{tabular}

We compare and analysis our grid based routing method and directed diffusion method which uses normal flooding method using lifetime and total energy consumption of sensor nodes. Fig. 9 shows the energy consumption of both two methods when two times of traffic centralization occurs. As you can see in the figure, grid method shows that traffic distribution is not centralized in specific nodes and is 
equally distributed than the normal flooding method. There is a difference between theoretical analysis model and real grid situation. It's because there are overheads, delays, and other difference in real transmission situation, such as small difference of Poisson distribution value occurred in Sleep or Idle mode. In real grid method, there is also another kind of energy consumption which occurs by table update, and it makes the difference of Fig. 9 even though it is not included in the energy consumption model. Fig. 10(a) shows how many nodes can be lost by time. As you can see in the figure, our grid method has more few numbers of lost nodes than the normal flooding method. In case of grid, there was no lost node during the transmission time for 100 times. By using grid method, we were able to reduce a part of energy consumption due to traffic centralization, but we were not able to reduce a part of energy consumption due to the node distribution which is centralized around sink or destination. If many nodes locate around destination, we can't reduce that kind of energy consumption of end nodes. Our grid method can reduce 1.2 to maximum 1.5 times of the number of lost nodes after initial node loss than flooding method. Fig. 10(b) shows the comparison of energy consumption rates between dense grid method and normal grid method. Dense grid method locates more nodes around sink and destination, and normal grid method locates nodes uniformly in the network area. Fig. 10(b) is a graph that shows statistics of the amount of consumed energy by the numbers of nodes per each transmission level.

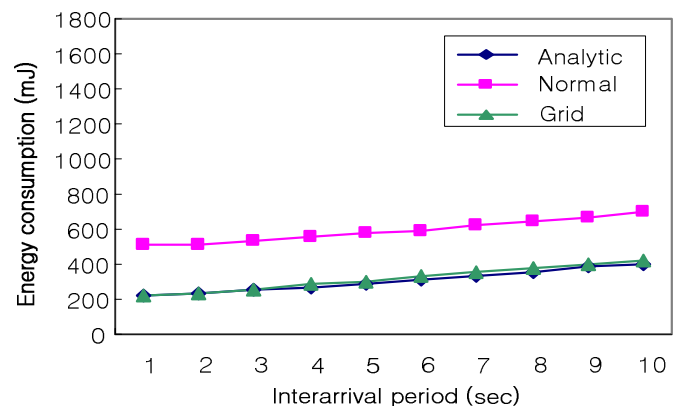

Fig. 9. Energy consumption of sensor nodes with normal flooding

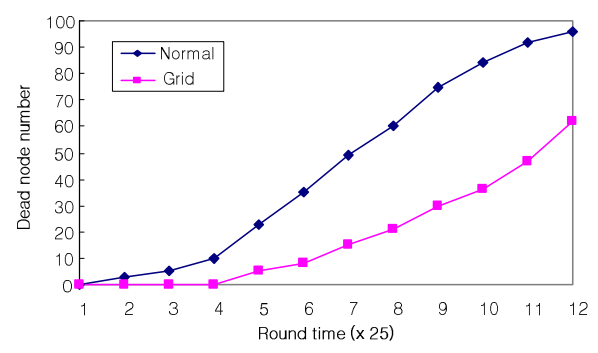

(a)

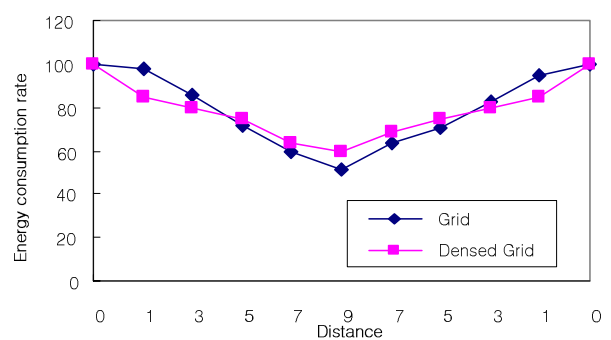

(b)

Fig. 10. Node loss number and energy consumption 
This simulation is processed in around $100 \times 100 \mathrm{~m}^{2}$, where transmission level is lower than $16^{\text {th }}$ step. As you can see in the figure, when we locate more sensor nodes in sink (left-side) or destination (right-side) of sensor network environment, the energy consumption rate of nodes in both ends is fallen little less. In case of grid method, traffic is distributed over every node. Therefore, energy exhaustion rate is regularly kept without energy centralization in each transmission level.

\section{Conclusions}

Grid computing approach proposed in this paper has different meaning with existing grid method. It does not use the method which uses lattice of existing grid method, but use the grid computing method which keeps effectiveness of total energy consumption regularly. This method also can be used with other routing methods. Transmission direction decision using transmission level can reduce unnecessary energy consumption through routing among nodes, and also increase lifetime of total network using energy level which prevents traffic concentration over specific nodes. Transmission is processed through energy critical value. Therefore, we can reduce many problems, such as accuracy reduction of processing or transmission for topology change due to node exhaustion. In conclusion, this grid method can be more suitable for environment which needs accurate transmission than real time transmission.

\section{References}

1. I.F. Akyildiz, W. Su, Y. Sank. and E. Cayirci, "A Survey on Sensor Networks," IEEE Communications Magazine, vol.40, no.8, pp. 102-114, August, 2002.

2. H.W. Tseng, S.H. Yang, P.Y. Chuang, E. H. Wu, and G.H. Chen, "An Energy Consumption Analytic Model for A Wireless Sensor MAC Protocol," IEEE 60th Vehicular Technology Conference, vol.6, pp. 4533-4537, September, 2004.

3. K. Sohrbi, J. Gao, V. Ailawadhi and G. J. Pottie, "Protocols for self-organization of a wireless sensor network," IEEE Personal Communications, vol. 7, no.5, pp. 16-27, 2000.

4. K. Akkays and M. Younis, "A Survey on Routing Protocols for Wireless Sensor Networks," Elsevier Ad Hoc Network Journal, vol.3, no.3, pp. 325-349, May, 2005.

5. T. V. Dam, K. Langendoen, "An adaptive energy efficient MAC protocol for wireless sensor networks," in Proceedings of ACM ENSS'2003, pp. 171-180, November, 2003.

6. H. Luo, F. Ye, J. Cheng, S.W. Lu and L. Zhang, "TTDD: Two-tier Data Dissemination in Large-scale Wireless Sensor Networks," ACM Wireless Networks, vol.11, no.1-2, 2005.

7. G.Kulkarni, C. Schurgers and M. B. Srivastava, "Dynamic Link Labels for Energy Efficient MAC Headers in Wireless Sensor Networks," IEEE International Conference on Sensors (Sensors'02), pp. 1520-1525, June, 2002.

8. J. Zhand and H. Shi, "Energy-efficient routing for 2D grid wireless sensor networks," Information Technology: Research and Education, Proceedings. ITRE2003, pp. 311-315, Aug. 2003.

9. M. Stemm and R.H Katz, "Measuring and reducing energy consumption of network interfaces in hand held Devices," IEICE Trans. on Comm., vol. E80-B, No. 8, 1997.

10. A. El-Hoiydi "Spatial TDMA and CSMA with Preamble Sampling for low power ad hoc Wireless sensor Networks," in Proceeding of the Seventh International Symposium on Computers and Communications(ISCC-'02), pp. 685-692, July, 2002. 